\title{
Mathematical analysis of Heat and mass transfer for Williamson nanofluid flow over an exponentially stretching surface subject to the exponential order surface temperature and heat flux
}

\author{
Zeeshan Badshah ${ }^{1}$, Rashid $\mathrm{Ali}^{2}$, M. Riaz Khan ${ }^{3, *}$ \\ *Corresponding author. E-mail: mrkhan.math@hotmail.com \\ ${ }^{1}$ Department of Mathematics, COMSATS Institute of Information Technology, Islamabad 44000, Pakistan \\ ${ }^{2}$ School of Mathematics and Statistics, Central South University, Changsha, Hunan 410083, PR China \\ ${ }^{3}$ LSEC and ICMSEC, Academy of Mathematics and Systems Science, Chinese Academy of Sciences; School of \\ Mathematical Science, University of Chinese Academy of Sciences, Beijing 100190, China
}

\begin{abstract}
This article investigates the features of heat and mass transfer for the steady two-dimensional Williamson nanofluid flow across an exponentially stretched surface depending on suction/injection. The boundary conditions incorporate the impacts of the Brownian motion and thermophoresis boundary. The analysis of heat transfer is carried out for the two cases of prescribed exponential order surface temperature (PEST) and prescribed exponential order heat flux (PEHF). The ongoing flow problem is mathematically modeled under the basic laws of motion and heat transfer. The similarity variables are allowed to transmute the governing equations of the problem into a similarity ordinary differential equation (ODEs). The solution of this reduced non-linear system of ODEs is supported by the Homotopy analysis method (HAM). The combination of HAM arrangements is acquired by plotting the h-curve. In order to evaluate the influence of several emergent parameters, the outcomes are presented numerically and are plotted diagrammatically as a consequence of velocity, temperature and concentration profiles.
\end{abstract}

Keywords: Heat transfer, Williamson fluid, Homotopy analysis method, exponential stretching, MHD, suction/injection.

Mathematics Subject Classification (2020): 74Axx, 76Bxx, 76Nxx, 65Nxx.

\section{Introduction}

Crane [1] was one of the early researchers who addressed the steady two-dimensional flow in terms of the Newtonian fluid, which was conducted by means of an elastic stretching sheet with a linearly varying velocity. The additional introductory judgment of the boundary layer flow across a stretched surface was donated by Sakiadis [2]. Wang [3] studied the Navier-Stokes equations as a function of time that were initially whiteuced to ODEs assisted by a dimensionless transformation and later solved through the shooting method. In accordance of the above concept, this modern research was further developed by a large number of researchers with the aim of considering different characteristics of flow and heat transfer that occurs in an infinite range of stretching surface. In these modern years, moderate importance has been established in the research of stretching boundary in view of its considerable and growing industrial technology applications containing sheet extraction, paper production, cooling of microchipping or metal film, hot rolling, bundle wrapping, and several others. As a result of above cases the ultimate compound of desired properties relies on the rate of cooling and the manner of stretching. In the context of these effective applications, significant work is carried out in multiple directions regarding the flow of the boundary layer and heat transfer by providing the surface stretching. The excess of research is provided in the publications about the steady flows of stretching phenomenon particularly in $[4,5]$. Although some effort has been conducted in terms of unsteady flows of stretching phenomenon $[6,7]$. Subsequently, the notion of the stretched surface was formulated by several other researchers [8-13] in various fluid models. 
Initially, the study of magneto-hydrodynamics (MHD) was reported in geophysical and astrophysical problems. During the last several years, this topic has come to the special focus based on their variety of applications in the medical, engineering, and petroleum-refining sectors. The existence of MHD in the nanofluid flow of threedimensional coordinate was planned by Sheikholeslami and Ellahi [14]. It was detected that the presence of MHD raises the resistive (drag) force and minimize the convection current. Additionally, the rate of heat transfer is visible to be developed. The thermo-physical properties of carbon nanotubes in MHD flow across a moving sheet have been addressed by Haq et al. [15]. It seems that the strength of the magnetic field escalates the fluid temperature. The channel flow of the rotating fluid describing the effect of the transversal magnetic field was recently studied by Mehmood et al. [16], which declares that the force of the magnetic field decays the wall flux. The oblique stagnation point flow with steady MHD forces was addressed by Borrelli et al. [17]. It was highlighted that if the strength of the electric field disappears, at that time the magnetic field occupies the flat plane of the stream rather than in the parallel directive to the flow. Additionally, the flow of the oblique stagnation point occurs solely when the applicable magnetic field is in the direction of dividing streamlines. Nadeem et al. [18] investigated the two-dimensional viscous flow of a nanofluid relating to the effect of the magnetic field across a curved surface. Some other studies regarding MHD flows are [19-22].

The existing research focuses on the two-dimensional steady Williamson nanofluid flow across an exponentially stretched surface depending on both suction as well as injection. The boundary conditions incorporate the impacts of the thermophoresis boundary and Brownian motion. The ongoing flow problem is mathematically modelled under the basic laws of motion and heat transfer. The similarity variables are allowed to transmute the governing equations of the problem into a non-linear ODEs. The solution of this reduced non-linear system of ODEs is supported by HAM. In order to assess the importance of several emergent parameters and variables, the outcomes are presented numerically and are plotted graphically.

\section{Mathematical Model}

Assume the two-dimensional steady Williamson nanofluid flow over a stretched exponential surface. It was recognized that the sheet is exponentially stretched with the varying velocity $U_{w}$ in the $x$-direction as well as the fluid which is occupied in $y$-direction is governed by the velocity $U_{w}$. Moreover, an exterior magnetic field of intensity $B_{0}$ is orthogonally placed to the direction of the stretched surface and the suction/injection phenomenon is signified by $v_{w}$. With these preconditions, the main boundary layer MHD equations for the continuity, momentum, energy, as well as concentration are respectively defined as [23-29].

$$
\begin{aligned}
& \frac{\partial u}{\partial x}+\frac{\partial u}{\partial y}=0 \\
& u \frac{\partial u}{\partial x}+v \frac{\partial u}{\partial x}=\nu \frac{\partial^{2} u}{\partial y^{2}}+\sqrt{2} \nu \Gamma \frac{\partial u}{\partial y} \frac{\partial^{2} u}{\partial y^{2}}-\sigma \frac{B_{0}^{2}}{\rho} u \\
& u \frac{\partial T}{\partial x}+v \frac{\partial T}{\partial y}=\alpha \frac{\partial^{2} u}{\partial y^{2}}+\frac{\left(\rho c_{p}\right)_{p}}{\left(\rho c_{p}\right)_{f}}\left[D_{B} \frac{\partial C}{\partial y} \frac{\partial T}{\partial y}+\frac{D_{T}}{T_{\infty}}\left(\frac{\partial T}{\partial y}\right)^{2}\right] \\
& u \frac{\partial C}{\partial x}+v \frac{\partial C}{\partial y}=\frac{D_{T}}{T_{\infty}}\left(\frac{\partial^{2} u}{\partial y^{2}}\right)+D_{B} \frac{\partial^{2} C}{\partial y^{2}}
\end{aligned}
$$

The boundary conditions connected to (1-4) are

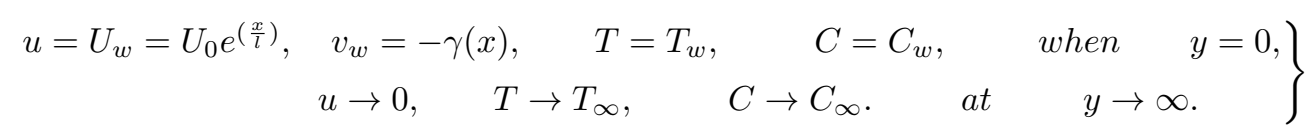

Here $u$ and $v_{w}$ individually mark the two parts of velocity in $x$ and $y$ path. Further, $\nu, \rho, \sigma, T, T$, $C$, $C_{\infty}$ separately provides the kinematic viscosity, density, electrical conductivity, temperature, ambient fluid temperature, concentration, and the nanoparticles volume fraction. Similarly, $\Gamma, \alpha,\left(\rho c_{p}\right)_{p},\left(\rho c_{p}\right)_{f}, D_{B}$ and $D_{T}$ delivers the shear stress, thermal diffusivity, heat capacity, fluid heat capacity, coefficient of Brownian and thermophoresis diffusion respectively. 


\section{Similarity solution of the governing equations}

The governing equations (1-4) are non-linear PDE's. We use the similarity transformation given below to convert the non-linear PDE's into a non-linear ODE's

$$
\left.\begin{array}{l}
u=U_{0} e^{\frac{x}{l}} f^{\prime}(\eta), \quad \eta=\sqrt{\frac{U_{0}}{2 \nu l}} y e^{\left(\frac{x}{2 l}\right)}, \\
v_{w}=-\sqrt{\frac{\nu U_{0}}{2 l}} e^{\frac{x}{2 l}}\left[f(\eta)+\eta f^{\prime}(\eta)\right] .
\end{array}\right\}
$$

The boundary conditions for the two cases of PEHF and PEST associated with the above equations (1-4) are given as

\section{PEST Case}

$$
T=T_{\infty}+\left(T_{w}-T_{\infty}\right) e^{\frac{x}{2 l}} \Theta(\eta), \quad g=\frac{C-C_{\infty}}{C_{w}-C_{\infty}}
$$

PEHF Case

$$
T=T_{\infty}+\frac{T_{w}-T_{\infty}}{K} e^{\frac{x}{2 l}} \sqrt{\frac{2 \nu l}{U_{0}}} \phi(\eta), \quad g=\frac{C-C_{\infty}}{C_{w}-C_{\infty}} .
$$

In view of the similarity transformation defined above, equation (1) fulfills in identical manner as well as equations (2-4) are reduced to the subsequent set of non-linear ODE's

$$
f^{\prime \prime \prime}-2 f^{\prime 2}+f f^{\prime \prime}+\lambda f^{\prime \prime} f^{\prime \prime \prime}-M f^{\prime}=0
$$

PEST Case

$$
\begin{aligned}
& \Theta^{\prime \prime}+P_{r}\left(f \Theta^{\prime}-f^{\prime} \Theta+N_{b} \Theta^{\prime} g^{\prime}+N_{t} \Theta^{\prime 2}\right)=0, \\
& g^{\prime \prime}+L_{e} P_{r}\left(f g^{\prime}\right)+\frac{N_{t}}{N_{b}} \Theta^{\prime \prime}=0
\end{aligned}
$$

PEHF Case

$$
\begin{aligned}
& \phi^{\prime \prime}+P_{r}\left(f \phi^{\prime}-f^{\prime} \phi+N_{b} \phi^{\prime} g^{\prime}+N_{t} \phi^{2}\right)=0, \\
& g^{\prime \prime}+L_{e} P_{r}\left(f g^{\prime}\right)+\frac{N_{t}}{N_{b}} \phi^{\prime \prime}=0
\end{aligned}
$$

Using the similarity transformation into boundary conditions (5), we obtain

$$
\left.\begin{array}{rl}
f=-v_{w}, & f^{\prime}=1, \quad \text { at } \quad \eta=0, \\
f^{\prime} \rightarrow 0, & \text { as } \quad \eta \rightarrow \infty .
\end{array}\right\}
$$

Boundary conditions for PEST case

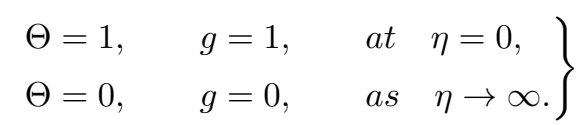

Boundary conditions for PEHF

$$
\left.\begin{array}{l}
\phi^{\prime}=-1, \quad g=1, \quad \text { at } \quad \eta=0, \\
\phi=0, \quad g=0, \quad \text { as } \quad \eta \rightarrow \infty .
\end{array}\right\}
$$

The similarity parameters appeared in above Eqs. (9-16) are $N_{t}, N_{b}, L_{e}, P_{r}, M, \lambda$ and $R^{e}$ which respectively represents the thermophoresis and Brownian motion parameter, Lewis number, Prandtl number, Hartmann number, Williamson parameter and the Reynolds number. These parameters are defined as

$$
\left.\begin{array}{c}
N_{t}=D_{B} \frac{(\rho c)_{p}}{(\rho c)_{f}}\left(C_{w}-C_{\infty}\right), \quad N_{b}=\frac{D_{T}}{T_{\infty}} \frac{(\rho c)_{p}}{(\rho c)_{f}} \frac{T_{w}-T_{\infty}}{\nu}, \quad L_{e}=\frac{\alpha}{D_{B}}, \\
M=-\frac{\sigma B_{0}}{U_{0} e^{\frac{x}{l}} \rho}, \quad \lambda=\left(\frac{U_{0}}{2}\right)^{\frac{2}{3}} \frac{\Gamma}{\sqrt{l}} e^{\frac{x}{2 l}}, \quad R_{e}=\frac{U L}{\nu} .
\end{array}\right\}
$$




\section{Solution by Homotopy Analysis Method}

We will use HAM to work out the equations (9-13) connected to the boundary conditions (14-16). This method requires an initial guess which is taken as

$$
f_{0}=1-v_{w}-e^{-\eta}, \quad \Theta_{0}=e^{-\eta}, \quad g_{0}=e^{-\eta}
$$

and the linear operators are

$$
\mathrm{Ł}_{1}(f)=f^{\prime \prime \prime}-f^{\prime}, \quad \mathrm{\iota}_{2}(\Theta)=\Theta^{\prime \prime}-\Theta, \quad \mathrm{\iota}_{3}(g)=g^{\prime \prime}-g
$$

These linear operator satisfy,

$$
\begin{aligned}
& \mathrm{E}_{1}\left[k_{1} e^{-\eta}+k_{2} e^{\eta}+k_{3}\right]=0 \\
& \mathrm{E}_{2}\left[k_{4} e^{-\eta}+k_{5} e^{\eta}\right]=0 \\
& \mathrm{E}_{3}\left[k_{6} e^{-\eta}+k_{7} e^{\eta}\right]=0
\end{aligned}
$$

where $k_{1}$ to $k_{7}$ are constants. Now we define the deformation of order zero as follows.

\section{PEST case}

$$
\begin{gathered}
(1-r) \mathrm{E}_{1}\left[\bar{f}(\eta, r)-f_{0}(\eta)\right]=r \hbar \bar{H}_{1} \tilde{N}_{1}[f(\bar{\eta}, r)] \\
(1-r) \mathrm{E}_{2}\left[\bar{\Theta}(\eta, r)-\Theta_{0}(\eta)\right]=r \hbar H_{2} \tilde{N}_{2}[\Theta(\bar{\eta}, r)] \\
(1-r) \mathrm{E}_{2}\left[\bar{g}(\eta, r)-g_{0}(\eta)\right]=r \hbar \bar{H}_{3} \tilde{N}_{3}[g(\bar{\eta}, r)],\left.\quad \bar{f}(\eta, r)\right|_{\eta=0}=-v_{w}, \\
\left.\bar{f}^{\prime}(\eta, r)\right|_{\eta=0}=1,\left.\bar{f}^{\prime}(\eta, r)\right|_{\eta=\infty}=0,\left.\quad \bar{\Theta}(\eta, r)\right|_{\eta=0}=1,\left.\bar{\Theta}(\eta, r)\right|_{\eta=\infty}=0 \\
\left.\bar{g}^{\prime}(\eta, r)\right|_{\eta=0}=1,\left.\bar{g}(\eta, r)\right|_{\eta=\infty}=0
\end{gathered}
$$

where $\hbar_{1}, \hbar_{2}, \hbar_{3}$ are auxiliary parameters which are non-zero and the auxiliary functions are $\bar{H}_{1}=\bar{H}_{2}=\bar{H}_{3}=1$. And

\section{PEST Case}

$$
\begin{aligned}
& \tilde{N}_{1}[\hat{f}(\eta, p)]=\frac{\partial^{3} f}{\partial \eta^{3}}-2 \frac{\partial f^{2}}{\partial \eta}+f \frac{\partial^{2} f}{\partial \eta^{2}}+\lambda \frac{\partial^{2} f}{\partial \eta^{2}} \frac{\partial^{2} f}{\partial \eta^{3}}-M \frac{\partial f}{\partial \eta} \\
& \tilde{N}_{2}[\tilde{\Theta}(\eta, r)]=\frac{\partial^{2} \theta}{\partial \eta^{2}}+P_{r}\left[f \frac{\partial \Theta}{\partial \eta}-\Theta \frac{\partial f}{\partial \eta}+N_{t}\left(\frac{\partial \Theta}{\partial \eta}\right)^{2}+N_{b} \frac{\partial g}{\partial \eta} \frac{\partial \Theta}{\partial \eta}\right] \\
& \tilde{N}_{3}[\tilde{g}(\eta, r)]=\frac{\partial^{2} g}{\partial \eta^{2}}+L_{e} P_{r}\left(f \frac{\partial g}{\partial \eta}\right)+\frac{N_{t}}{N_{b}} \frac{\partial^{2} \Theta}{\partial \eta^{2}} \\
& \hat{f}(\eta, 0)=f_{0}(\eta), \quad \hat{f}(\eta, 1)=f(\eta), \\
& \hat{\Theta}(\eta, 0)=\Theta_{0}(\eta), \quad \hat{\Theta}(\eta, 1)=\Theta(\eta), \\
& \hat{g}(\eta, 0)=g_{0}(\eta), \quad \hat{g}(\eta, 1)=g(\eta),
\end{aligned}
$$

Where $r$ is the embedding parameters which varies from 0 to 1 and the initial solution $f_{0}(\eta)$ varies to the final solution $f(\eta), \Theta_{0}(\eta)$ to $\left.\Theta_{(} \eta\right)$, and $g_{0}(\eta)$ to $g(\eta)$. 
Using Taylor series we have

\section{PEST Case}

$$
\begin{aligned}
& \hat{f}(\eta, r)=f_{0}(\eta)+\sum_{m=1}^{\infty} r^{m} f_{m}(\eta) \\
& \hat{\Theta}(\eta, r)=\Theta_{0}(\eta)+\sum_{m=1}^{\infty} r^{m} \Theta_{m}(\eta) \\
& \hat{g}(\eta, r)=g_{0}(\eta)+\sum_{m=1}^{\infty} r^{m} g_{m}(\eta), \\
& \hat{f_{m}}(\eta)=\left.\frac{1}{m !} \frac{\partial^{m} f(\hat{\eta}, r)}{\partial r^{m}}\right|_{r=0}, \\
& \hat{\Theta_{m}}(\eta)=\left.\frac{1}{m !} \frac{\partial^{m} \Theta(\hat{\eta}, r)}{\partial r^{m}}\right|_{r=0}, \\
& \hat{g_{m}}(\eta)=\left.\frac{1}{m !} \frac{\partial^{m} g(\hat{\eta}, r)}{\partial r^{m}}\right|_{r=0} .
\end{aligned}
$$

The $m$ th-order problem will be like follow.

$$
\begin{aligned}
& \mathrm{Ł}_{1}\left[f_{m}(\eta)-X_{m} f_{m-1}(\eta)\right]=\hbar_{1} \check{R}_{m}^{1}(\eta) \\
& \mathrm{E}_{2}\left[\Theta_{m}(\eta)-X_{m} \Theta_{m-1}(\eta)\right]=\hbar_{2} \check{R}_{m}^{2}(\eta), \\
& \mathrm{Ł}_{3}\left[g_{m}(\eta)-X_{m} g_{m-1}(\eta)\right]=\hbar_{3} \check{R}_{m}^{3}(\eta), \\
& f_{m}(0)=f_{m}^{\prime}(0)=f_{m}^{\prime}(\infty)=0, \\
& \Theta_{m}(0)=\Theta_{m}(\infty)=0, \\
& g_{m}^{\prime}(0)=g_{m}(\infty)=0 \text {. } \\
& X_{m}= \begin{cases}0, & m \leq 1 \\
1, & m>1\end{cases} \\
& \check{R}_{m}^{1}(\eta)=f_{m-1}^{\prime \prime \prime}(\eta)+\sum_{k=0}^{m-1} f_{m-1-k} f_{k}^{\prime \prime}-2 \sum_{k=0}^{m-1} f_{m-1-k}^{\prime} f_{k}^{\prime} \\
& \check{R}_{m}^{2}(\eta)=\Theta_{m-1}^{\prime \prime}+\operatorname{Pr} \sum_{k=0}^{m-1}\left[f_{m-1-k} \Theta_{k}-f_{m-1-k}^{\prime} \Theta_{k}+N_{b} \Theta_{m-1-k}^{\prime} g_{k}^{\prime}+N_{t} \Theta_{k}^{\prime} \Theta_{m-1-k}^{\prime}\right] \text {, } \\
& \check{R}_{m}^{3}(\eta)=g_{m-1}^{\prime \prime}=L_{e} P_{r} \sum_{k=0}^{m-1}\left[f_{m-1-k} g_{k}^{\prime}-f_{m-1-k}^{\prime} g_{k}-f_{m-1-k}^{\prime} g_{k}^{\prime}-\right]+\frac{N_{t}}{N_{b}} \Theta_{m-1}^{\prime \prime} \text {. }
\end{aligned}
$$

Employing the mathematica we get the solution

$$
\begin{aligned}
& f(\eta)=\sum_{m=0}^{\infty} f_{m}(\eta)=\lim _{M \rightarrow \infty}\left[\sum_{m=0}^{M} b_{m, 0}^{0}+\sum_{\eta=1}^{M+1} e^{-n \eta}\left(\sum_{m=\eta-1}^{M} \sum_{k=0}^{m+1-\eta} b_{m, \eta}^{k} \eta^{k}\right)\right] \\
& \Theta(\eta)=\sum_{m=0}^{\infty} \Theta_{m}(\eta)=\lim _{M \rightarrow \infty}\left[\sum_{\eta=1}^{M+2} e^{-n \eta}\left(\sum_{m=\eta-1}^{M+1} \sum_{k=0}^{m+1-\eta} B_{m, \eta}^{k} \eta^{k}\right)\right] \\
& g(\eta)=\sum_{m=0}^{\infty} g_{m}(\eta)=\lim _{M \rightarrow \infty}\left[\sum_{\eta=1}^{M+2} e^{-n \eta}\left(\sum_{m=\eta-1}^{M+1} \sum_{k=0}^{m+1-\eta} G_{m, \eta}^{k} \eta^{k}\right)\right]
\end{aligned}
$$




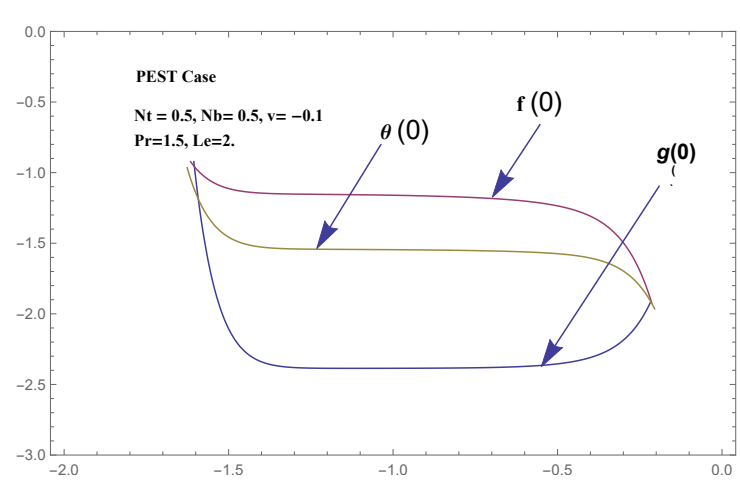

Figure 1: h-curves for velocity, temperature and concentration profile for PEST.

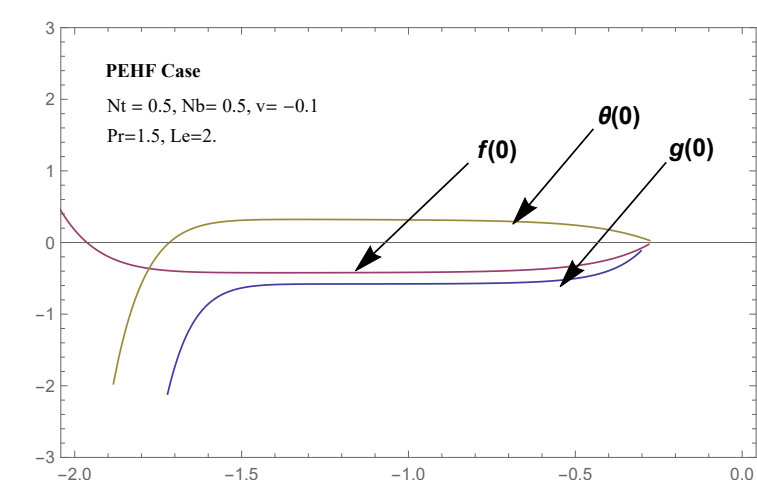

Figure 2: h-curves for velocity, temperature and concentration profile for PEHF.

Table 1: Convergence of series solution for different order of approximation for various values of $P_{r}=2$, $N_{b}=0.5, N_{t}=0.2, L_{e}=2, v=0.2$.

\begin{tabular}{cccc}
\hline Order of approximation & $f^{\prime \prime}(0)$ & $g^{\prime}(0)$ & $\Theta^{\prime}(0)$ \\
\hline 1 & -1.36 & -0.49 & -0.85 \\
5 & -1.44442 & -0.358085 & -0.909949 \\
10 & -1.450456 & -0.352815 & 0.907993 \\
17 & -1.45116 & -0.353012 & -0.908089 \\
22 & -1.45117 & -0.353009 & -0.908084 \\
24 & -1.45117 & -0.35301 & 908084 \\
26 & -1.45117 & -0.45301 & -0.908084 \\
27 & -1.45117 & -0.45301 & -0.908084 \\
28 & -1.45117 & -0.45301 & -0.908084 \\
29 & -1.45117 & -0.45301 & -0.908084 \\
\hline
\end{tabular}

where $b_{m, 0}^{0}, b_{m, 0}^{k}, B_{m, n}^{k}, G_{m, n}^{k}$ are constants.

$\mathrm{PHEF}$ case is solved in a similar way.

\section{Convergence of solution}

The region for which the solution of momentum, energy and concentration equations converge, is obtained by plotting h curves. The range for auxiliary parameter $\hbar$ are.

\section{PEST Case}

$$
\begin{aligned}
& -1.3 \leq \hbar_{f} \leq-0.7 \\
& -1.3 \leq \hbar_{g} \leq-0.6 . \\
& -1.3 \leq \hbar_{\Theta} \leq-0.5 .
\end{aligned}
$$

\section{PEHF Case}

$$
\begin{aligned}
& -1.3 \leq \hbar_{f} \leq-0.8 \\
& -1.3 \leq \hbar_{g} \leq-0.8 \\
& -1.4 \leq \hbar_{\Theta} \leq-0.6 .
\end{aligned}
$$

h-curves described the rang of parameter $\hbar$ for which the solution $f, g$, and $\Theta$ are converge. 


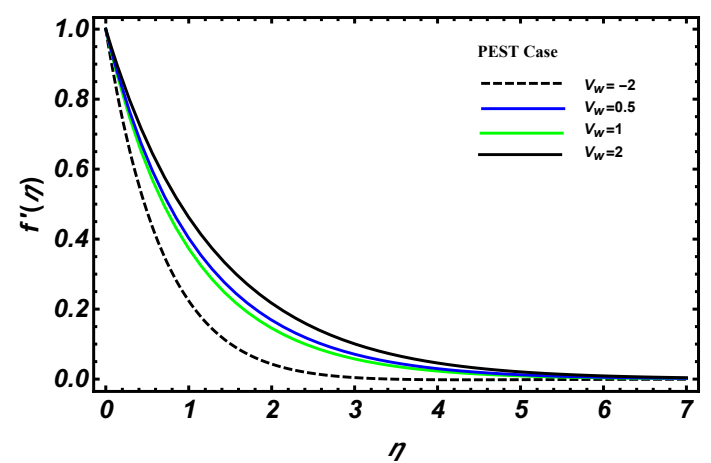

Figure 3: velocity for different values of suction/injection parameter for PEST.

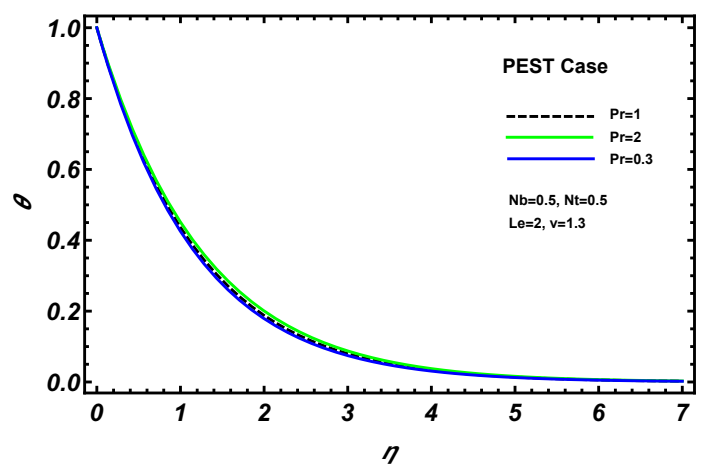

Figure 5: PEST for different values of $P_{r}$ number.

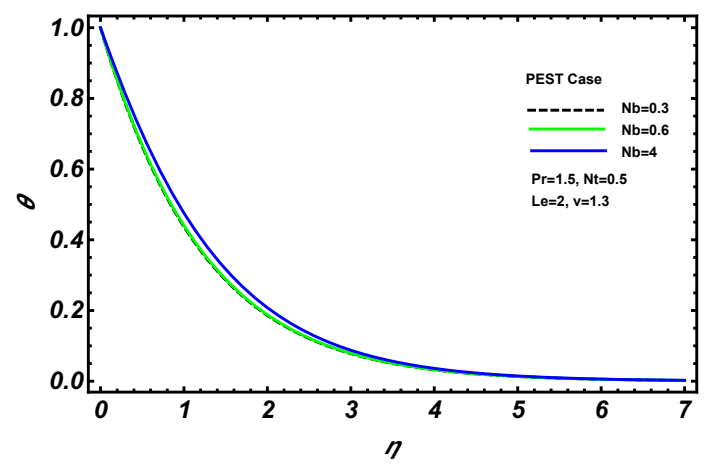

Figure 7: PEST for different values of $N_{b}$ number.

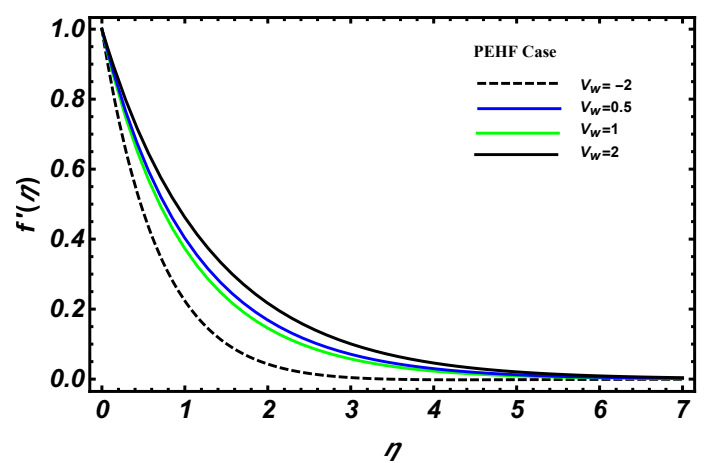

Figure 4: velocity for different values of suction/injection parameter for PEHF.

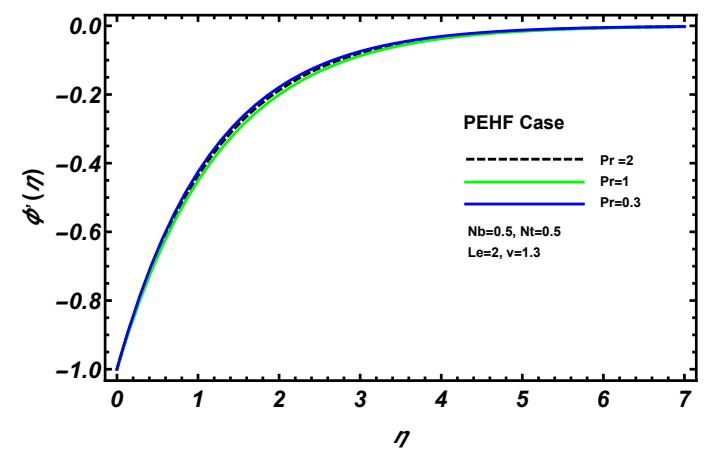

Figure 6: PEHF for different values of $P_{r}$ number.

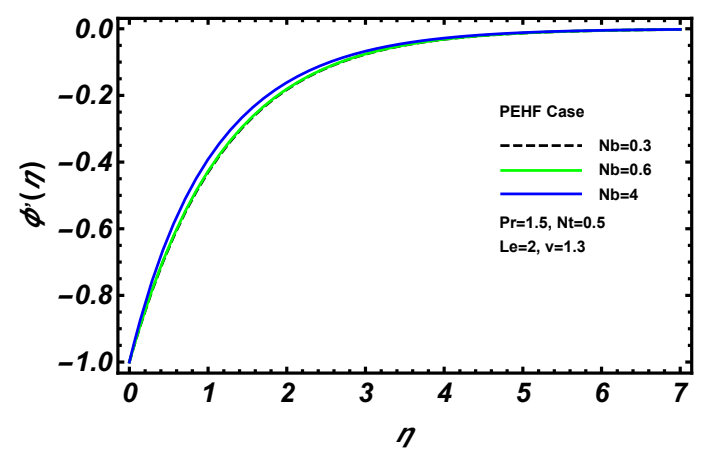

Figure 8: PEHF for different values of $N_{b}$ number 


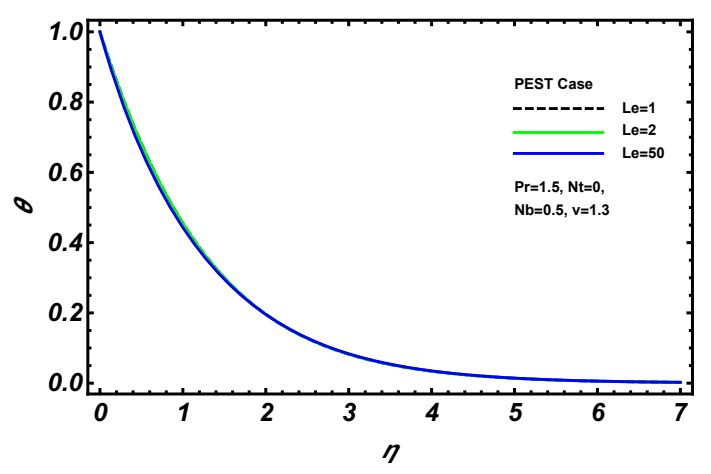

Figure 9: PEST for different values of $L_{e}$ number.

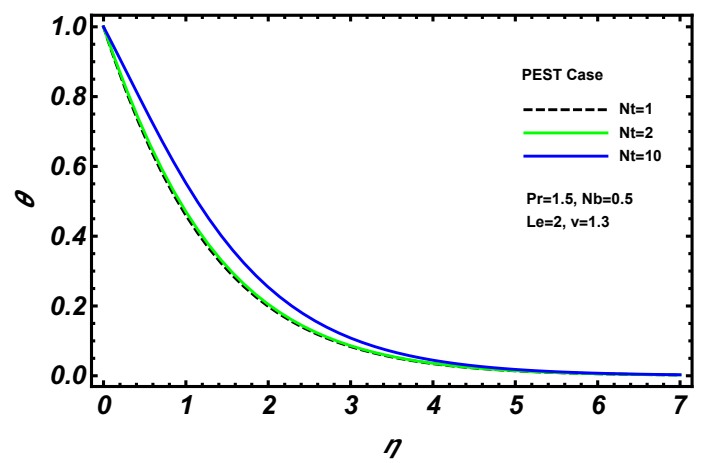

Figure 11: PEST for different values of $N_{t}$ number.

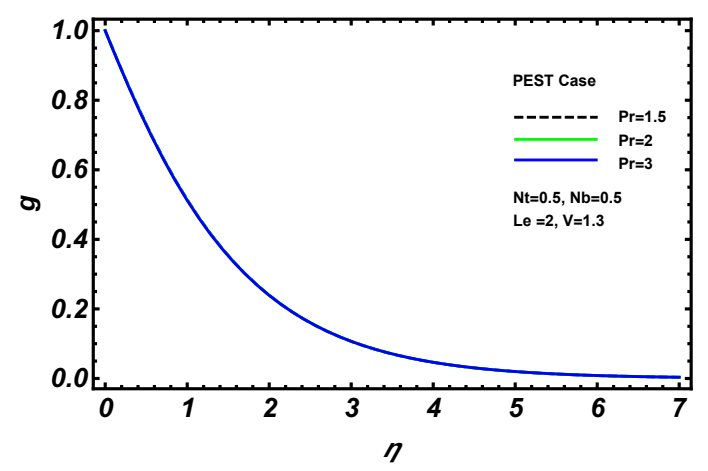

Figure 13: PEST for different values of $P_{r}$ number.

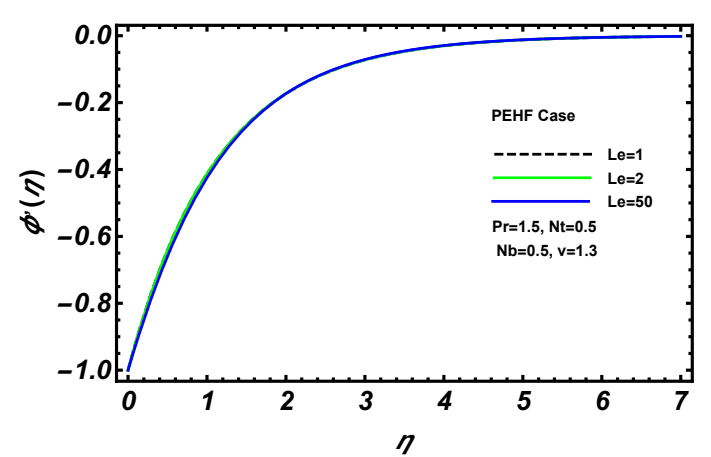

Figure 10: PEHF for different values of $L_{e}$ number.

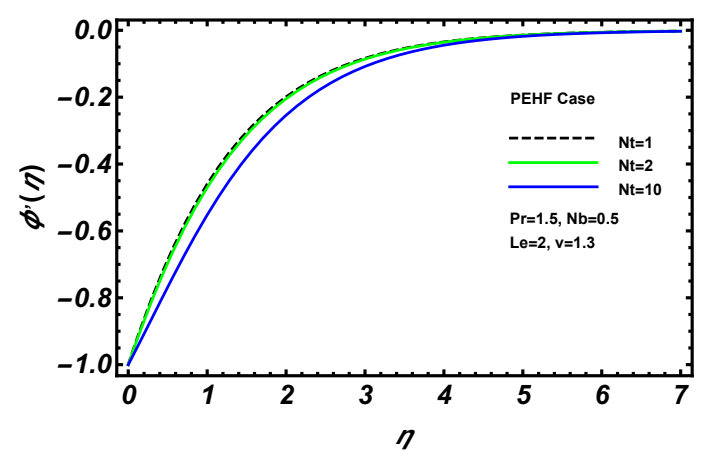

Figure 12: PEHF for different values of $N_{t}$ number.

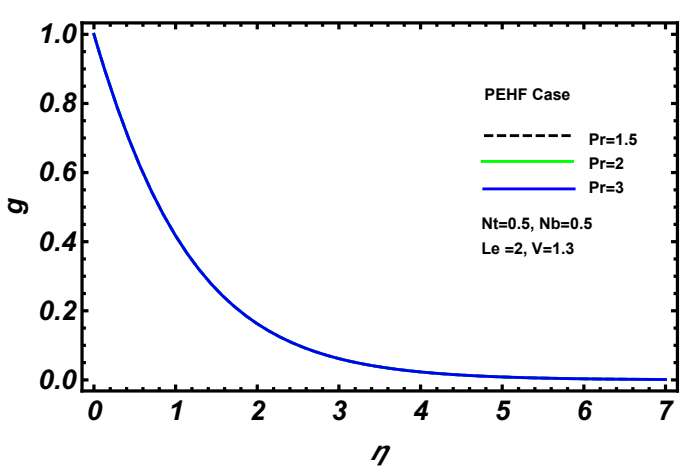

Figure 14: PEHF for different values of $P_{r}$ number. 


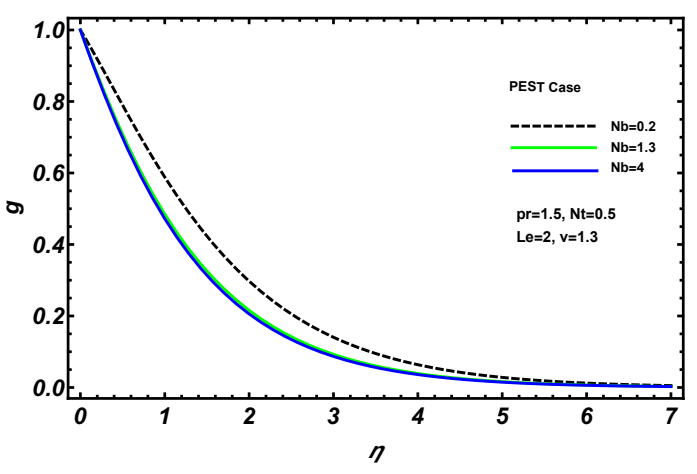

Figure 15: PEST for different values of $N_{b}$ number.

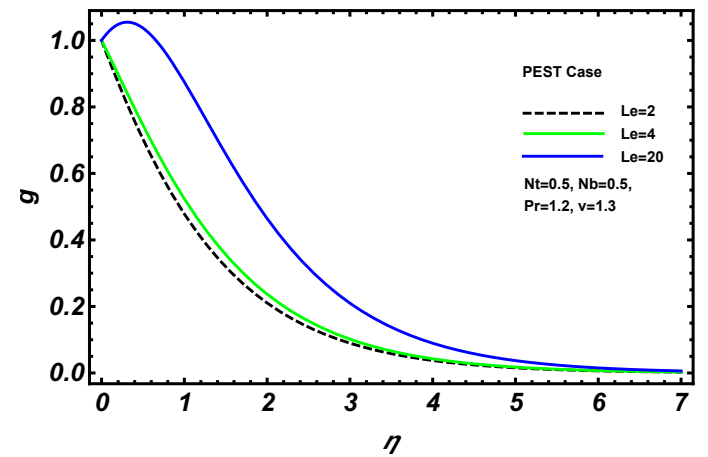

Figure 17: PEST for different values of $L_{e}$ number.

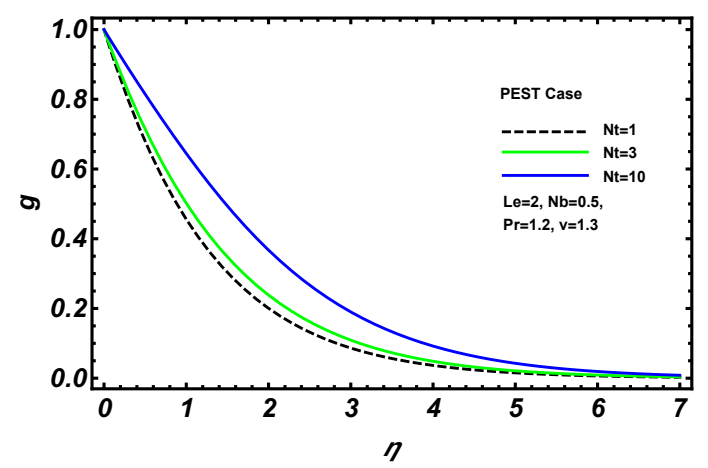

Figure 19: PEST for different values of $N_{t}$ number.

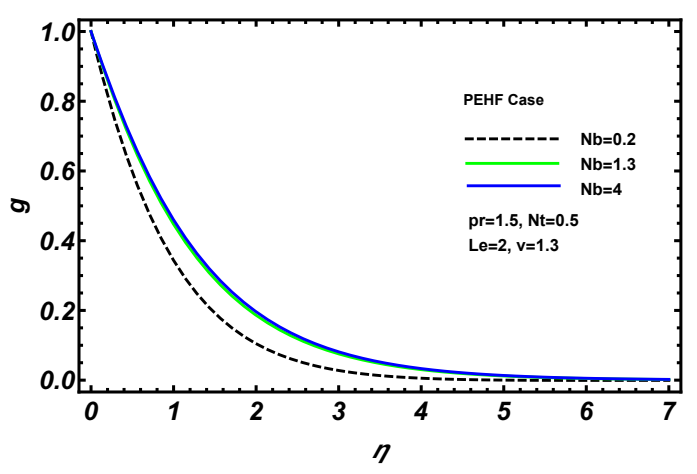

Figure 16: PEHF for different values of $N_{b}$ number.

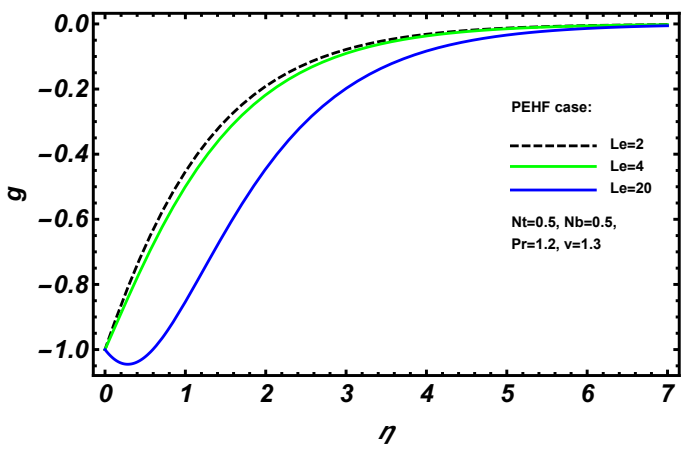

Figure 18: PEHF for different values of $L_{e}$ number.

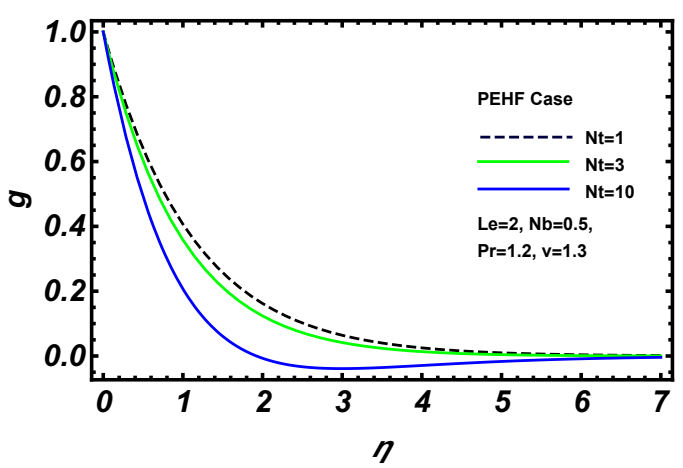

Figure 20: PEHF for different values of $N_{t}$ number. 


\section{Results and Discussion}

The solution of the governing problem is executed with the help of Mathematica and the results are plotted graphically, which characterizes the behavior of the problem. A comparison is made between the graphical results of two distinct cases of the problem i.e. PEHF and PEST. Initially we have prepared the results of h-curves for velocity, temperature and concentration profiles as plotted in figures $\mathbf{1} \& \mathbf{2}$. To deal with different flow parameters we have first prepared the individual graphical results of velocity profile for the two different classes of PEST and PEHF as shown in figures 3 \& 4 . These figures illustrate the effect of suction/injection parameter, which is in direct relation to the velocity profile, i.e. when we increase the suction/injection parameter the velocity profile also going to increase for both PEST and PEHF in the same manner. In the same way, the temperature profiles are plotted for distinct values of the Prandtl number as found in figures $\mathbf{5} \& \mathbf{6}$. It is noticed that with the escalation $P_{r}$, the temperature profile shoots up only for PEST case, whereas temperature decrease in the case of PEHF.

In figures $\mathbf{7} \& \mathbf{8}$ the different values of $N_{b}$ have shown their influence on the $\Theta(\eta)$. It appears that with the escalation of $N_{b}$ there is a boost in $\Theta(\eta)$ for both PEST as well as PEHF case and the boundary layer declines for PEST, although it modifies for PEHF. Figures 9 \& 10 indicates the $\Theta(\eta)$ profile for the different values of $L_{e}$ which provides a very minimal change in $\Theta(\eta)$ for each case of PEST and PEHF. However, by making a big change in $L_{e}$ there is a slight decrease in the $\Theta(\eta)$ for each case. For the various values of $N_{t}$, the separate graphical results for the mutual case of PEST and PEHF can be detected respectively in figures 11 \& 12. It appears that the $\Theta(\eta)$ boosts with the escalation of $N_{t}$ used for the PEST situation and for PEHF conditions $\Theta(\eta)$ decreases.

The behavior of concentration profile for different values of physical parameters $P_{r}$ is respectively shown in figures $13 \& 14$. These figures signify the change in nano-particles volume fraction which is caused by making the change in $P_{r}$ number. One can see that, for the joint conditions of PEST and PEHF, there is a negligible change in $g$ for different values of $P_{r}$ as well as the same behavior is detected for the boundary layer. Similarly, the concentration profile for several values of $N_{b}$ is shown in figures $15 \& \mathbf{1 6}$. We have noted that the escalation of $N_{b}$, induces a decrease in concentration profile $g$ for PEST conditions, although $g$ increases for PEHF conditions. The effect of $L_{e}$ number on the nano-particles volume fractions is shown in figures $\mathbf{1 7}$ \& 18. We see the same behavior for the joint conditions of PEST and PEHF, that is with the increase of $L_{e}$ number the concentration $g$ individually shoots up for PEST as well as PEHF conditions. Figures 19 \& 20. represent the effect of $N_{t}$ on $g$. It appears that with the escalation of $N_{t}$, there is an increase in $g$ for PEHF case whereas reduction is detected in case of PEST case.

\section{Conclusion}

- The velocity profile modifies for the enhancement of suction/injection parameter in both PEST and PEHF case.

- The temperature profile modifies for the enhancement of $P_{r}$ only for PEST case, whereas it declines in the case of PEHF.

- The escalation of $N_{b}$ values causes to boost the temperature profile for both PEST as well as PEHF case while.

- The escalation of $N_{t}$ values causes to improve both the temperature profile and concentration profile only in case of PEST whereas a reduction is detected in case of PEST for both profiles.

- The escalation of $N_{b}$ causes a decrease in concentration profile $g$ for PEST case, although $g$ increases for PEHF case.

- The escalation of $L_{e}$ number causes to increase the nanoparticles volume fraction for both PEST and PEHF case. 


\section{References}

[1] Crane LJ. Flow past a stretching plate. Zeitschrift für angewandte Mathematik und Physik ZAMP. 1970;21(4):645-647.

[2] Sakiadis BC. Boundary-layer behavior on continuous solid surfaces: II. The boundary layer on a continuous flat surface. AiChE journal. 1961;7(2):221-225.

[3] Wang CY. Liquid film on an unsteady stretching surface. Quarterly of Applied Mathematics. 1990;48(4):601-610.

[4] Li X, Khan AU, Khan MR, Nadeem S, Khan SU. Oblique stagnation point flow of nanofluids over stretching/shrinking sheet with Cattaneo-Christov heat flux model: existence of dual solution. Symmetry. 2019;11(9):1070.

[5] Khan MR. Numerical analysis of oblique stagnation point flow of nanofluid over a curved stretching/shrinking surface. Physica Scripta. 2020;9(10):105704.

[6] Nadeem S., Khan AU. MHD oblique stagnation point flow of nanofluid over an oscillatory stretching/shrinking sheet: Existence of dual solutions. Physica Scripta. 2019;94(7):075204.

[7] Nadeem S, Ullah N, Khan AU, Akbar T. Effect of homogeneous-heterogeneous reactions on ferrofluid in the presence of magnetic dipole along a stretching cylinder. Results in physics. 2017;7:3574-3582.

[8] Sahoo B. Effects of slip, viscous dissipation and Joule heating on the MHD flow and heat transfer of a second-grade fluid past a radially stretching sheet. Applied Mathematics and Mechanics (English Edition). 2010;31(2):159-173.

[9] Qaiser D, Zheng Z, Khan MR. Numerical assessment of mixed convection flow of Walters-B nanofluid over a stretching surface with Newtonian heating and mass transfer. Thermal Science and Engineering Progress. 2020;100801.

[10] Hayat T, Asad S, Alsaedi A. Flow of variable thermal conductivity fluid due to inclined stretching cylinder with viscous dissipation and thermal radiation. Applied Mathematics and Mechanics (English Edition). 2014;35(6):717-728.

[11] Meqahid AM. Carreau fluid flow due to nonlinearly stretching sheet with thermal radiation, heat flux, and variable conductivity. Applied Mathematics and Mechanics (English Edition). 2019;40(11):1615-1624.

[12] Zhu J, Zheng L, Zhang X. Second-order slip MHD flow and heat transfer of nanofluids with thermal radiation and chemical reaction. Applied Mathematics and Mechanics (English Edition). 2015;36(9):11311146.

[13] Mahanthesh B, Gireesha BJ, Shehzad SA, Abbasi FM, Gorla RS. Nonlinear three-dimensional stretched flow of an Oldroyd-B fluid with convective condition, thermal radiation, and mixed convection. Applied Mathematics and Mechanics (English Edition). 2017;38(7):969-980.

[14] Sheikhlesami M, Ellahi R. Three-dimensional mesoscopic simulation of magnetic field effect on natural convection of nanofluid. International Journal of Heat and Mass Transfer. 2015;89:799-808.

[15] Haq RU., Khan ZH, Khan WA. Thermophysical effects of carbon nanotubes on MHD flow over a stretching surface. Physica E: Low-dimensional Systems and Nanostructures. 2014;63:215-222.

[16] Mehmood R, Nadeem S, Massod S. Effects of transverse magnetic field on a rotating micropolar fluid between parallel plates with heat transfer. Journal of Magnetism and Magnetic Materials. 2016;40:10061014.

[17] Borrelli A, Giantesio G, Patria MC. MHD oblique stagnation-point flow of a Newtonian fluid. Zeitschrift für angewandte Mathematik und Physik. 2012;63(2):271-294.

[18] Nadeem S, Khan MR, Khan AU. MHD stagnation point flow of viscous nanofluid over a curved surface. Physica Scripta. 2019;94(11):115207.

[19] Khan M, Malik R, Anjum A. Exact solutions of MHD second Stokes flow of generalized Burgers fluid. Applied Mathematics and Mechanics (English Edition). 2015;36(2):211-224. 
[20] Ali FM, Nazar R, Arifin NM, Pop I. MHD stagnation-point flow and heat transfer towards stretching sheet with induced magnetic field. Applied Mathematics and Mechanics (English Edition). 2011;32(4):409-418.

[21] Zhu J, Zheng LC, Zhang ZG. Effects of slip condition on MHD stagnation-point flow over a power-law stretching sheet. Applied Mathematics and Mechanics (English Edition). 2010;31(4):439-448.

[22] Rauf A, Abbas Z, Shehzad S A. Utilization of Maxwell-Cattaneo law for MHD swirling flow through oscillatory disk subject to porous medium. Applied Mathematics and Mechanics (English Edition). 2019;40(6):837-850.

[23] Haq RU, Nadeem S, Khan ZH, Akbar NS. Thermal radiation and slip effects on MHD stagnation point flow of nanofluid over a stretching sheet. Physica E: Low-dimensional systems and nanostructures. 2015;65:1723.

[24] Ramzan M, Chung JD, Ullah N. Partial slip effect in the flow of MHD micropolar nanofluid flow due to a rotating disk-A numerical approach. Results in physics. 2017;7:3557-3566.

[25] Nadeem S, Akhtar S, Abbas N. Heat transfer of Maxwell base fluid flow of nanomaterial with MHD over a vertical moving surface. Alexandria Engineering Journal. 2020.

[26] Uddin I, Ullah I, Ali R, Khan I, Nisar KS. Numerical analysis of nonlinear mixed convective MHD chemically reacting flow of Prandtl-Eyring nanofluids in the presence of activation energy and Joule heating. Journal of Thermal Analysis and Calormitory. 2020. https://doi.org/10.1007/s10973-020-095742.

[27] Ullah I, Ali R, Khan I, Nisar KS. Insight into kerosene conveying SWCNT and MWCNT nanoparticles through a porous medium: significance of Coriolis force and entropy generation. Physica Scripta. 2021;1:111.

[28] Nadeem S, Hussain ST. Heat transfer analysis of Williamson fluid over exponentially stretching surface. Applied Mathematics and Mechanics (English Edition). 2014;35(4):489-502.

[29] Nadeem S, Hussain ST, Lee C. Flow of a Williamson fluid over a stretching sheet. Brazilian journal of chemical engineering. 2013;30(3):619-625. 